\title{
Baicalein induces apoptosis via ROS-dependent activation of caspases in human bladder cancer 5637 cells
}

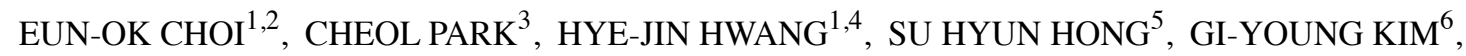 \\ EUN-JU CHO ${ }^{2}$, WUN-JAE $\mathrm{KIM}^{7}$ and YUNG HYUN CHOI ${ }^{1,5}$ \\ ${ }^{1}$ Anti-Aging Research Center, Dongeui University, Busan 614-714; ${ }^{2}$ Department of Food and Nutrition, \\ College of Human Ecology, Pusan National University, Busan 609-735; Departments of ${ }^{3}$ Molecular Biology and \\ ${ }^{4}$ Food and Nutrition, College of Natural Sciences and Human Ecology, Dongeui University, Busan 614-714; \\ ${ }^{5}$ Department of Biochemistry, Dongeui University College of Korean Medicine, Busan 614-052; \\ ${ }^{6}$ Laboratory of Immunobiology, Department of Marine Life Sciences, Jeju National University, Jeju 690-756; \\ ${ }^{7}$ Department of Urology, Chungbuk National University College of Medicine, Cheongju 361-763, Republic of Korea
}

Received May 16, 2016; Accepted July 1, 2016

DOI: 10.3892/ijo.2016.3606

\begin{abstract}
Baicalein is a flavonoid derived originally from the root of Scutellaria baicalensis Georgi, which has been used in Oriental medicines for treating various diseases. Although this compound has been reported to have anticancer activities in several human cancer cell lines, the therapeutic effects of baicalein on human bladder cancer and its mechanisms of action have not been extensively studied. This study investigated the proapoptotic effects of baicalein in human bladder cancer 5637 cells. For this study, cell viability and apoptosis were evaluated using the 3-(4,5-dimethylthia-zol2-yl)-2,5-diphenyltetrazolium bromide assay, trypan blue dye exclusion assay 4,6-diamidino-2-phenylindole staining, and flow cytometry. Measurements of the mitochondrial membrane potential (MMP), caspase activity assays and western blots were conducted to determine whether 5637 cell death occurred by apoptosis. Treatment with baicalein resulted in a concentration-dependent growth inhibition coupled with apoptosis induction, as indicated by the results of nuclei morphology examination and flow cytometry analyses. The induction of the apoptotic cell death of 5637 cells by baicalein exhibited a correlation with the downregulation of members
\end{abstract}

Correspondence to: Professor Wun-Jae Kim, Department of Urology, Chungbuk National University, College of Medicine and Institute for Tumor Research, 7761 Sunhwan-ro, Seowon-gu, Cheongju 362-763, Republic of Korea

E-mail:wjkim@chungbuk.ac.kr

Professor Yung Hyun Choi, Department of Biochemistry, Dongeui University College of Korean Medicine, 52-57 Yangjeong-ro, Busanjin, Busan 614-052, Republic of Korea

E-mail: choiyh@deu.ac.kr

Key words: baicalein, bladder cancer 5637 cells, apoptosis, caspase, reactive oxygen species of the inhibitor of apoptosis protein (IAP) family, including cIAP-1 and cIAP-2, and the activation of caspase- 9 and -3 accompanied by proteolytic degradation of poly(ADP-ribose)polymerase. The study also showed that baicalein decreases the expression of the proapoptotic protein Bax, increases antiapoptotic Bcl-2 expression, and noticeably aggravates the loss of MMP. Concomitantly, the data showed that baicalein increases the levels of death receptors and their associated ligands and enhances the activation of caspase- 8 and truncation of Bid. However, the pan-caspase inhibitor can reverse baicalein-induced apoptosis, demonstrating that it is a caspasedependent pathway. Moreover, it was found that baicalein can induce the production of reactive oxygen species (ROS) and that pretreatment with the antioxidant $\mathrm{N}$-acetyl-L-cysteine significantly attenuates the baicalein effects on the loss of MMP and activation of caspase. In addition, the blocking of ROS generation decreases the apoptotic activity and antiproliferative effect of baicalein, indicating that baicalein induces apoptosis of 5637 cells through the ROS-dependent activation of caspases.

\section{Introduction}

Bladder cancer is the most prevalent tumor of the urinary tract worldwide. The majority of bladder cancers are low-grade noninvasive tumors that may progress to the invasive phenotype. In contrast to non-invasive bladder cancer, muscle invasive tumors tend to metastasize to other organs and have a very poor prognosis $(1,2)$. Recent epidemiological studies have shown that the incidence of bladder cancer is highest in the developed countries of Western Europe, North America, and Australia and lowest in Asian countries, which may be connected with their different diets and living habits $(3,4)$. Although radiation therapy, adjuvant chemotherapy, and combinations of these modalities are standard options for managing bladder cancer, current treatments of bladder cancer have high recurrence rates and may cause strong side effects $(5,6)$. Therefore, the discovery of effective anticancer drug candidates for the treat- 
ment of bladder cancer is still required for the advancement of the medical treatment of bladder cancer patients.

Recently, natural products or compounds, such as plantderived products, have been recognized as powerful resources for new cancer drug discovery, because they may reduce adverse side effects $(7,8)$. Baicalein $(5,6,7$-trihydroxyflavone) is one of the major phenolic flavonoids isolated from the rhizome of Scutellaria baicalensis Georgi $(9,10)$, which has been widely used in traditional Oriental medicine for treating various inflammatory diseases, chronic hepatitis, bacterial and viral infections, allergies, and ischemia $(11,12)$. Recent research on baicalein has documented a wide spectrum of therapeutic properties, including antimicrobial, anti-inflammatory, antioxidative, immunomodulatory, and antiangiogenesis effects (10,12-15). Several studies have indicated that baicalein exhibits anticancer activities due to its ability to inhibit cell growth and to induce cell cycle arrest at the G1 phase and apoptotic pathways in distinct cancer cells (16-20). Interestingly, this agent does not exert an apoptotic effect on normal cells and therefore can be developed as an anticancer drug (21-23). Moreover, we recently demonstrated that baicalein causes apoptosis in human lung carcinoma cells by changing the apoptotic gene expressions through reactive oxygen species (ROS) generation (24).

However, the direct molecular target and mode of the baicalein anticancer mechanism in human bladder cancer cells have not been fully clarified. Therefore, this study used human muscle invasive bladder cancer 5637 cells to identify additional molecular mechanisms supporting the antiproliferative and apoptotic effects of baicalein.

\section{Materials and methods}

Chemicals and antibodies. RPMI-1640 medium, fetal bovine serum (FBS), and antibiotics were purchased from Welgene (Daegu, Korea). Baicalein (purity 98\%,Fig. 1A), 4,6-diamidino2-phenylindole (DAPI), 3-(4,5-dimethyl-2-thiazolyl)-2, 5-diphenyl-2H-tetrazolium (MTT), and N-acetyl-L-cysteine (NAC) were purchased from Sigma-Aldrich Chemical Co. (St. Louis, MO, USA). N-benzyloxycarbonyl-Val-Ala-Aspfluoromethylketone (z-VAD-fmk), a pan-caspase inhibitor, and 5,5',6,6'-tetrachloro-1,1',3,3'-tetraethyl-imidacarbocyanine iodide (JC-1) were purchased from Calbiochem (San Diego, CA, USA). 2',7'-dichlorofluorescein diacetate (DCF-DA) and fluorescein-conjugated Annexin V (Annexin V-FITC) were obtained from Molecular Probes (Eugene, OR, USA) and BD Biosciences Pharmingen (San Jose, CA, USA), respectively. An enhanced chemiluminescence (ECL) detection system and in vitro caspase colorimetric assay kits were purchased from Amersham Corp. (Arlington Heights, IL, USA) and R\&D Systems (Minneapolis, MN, USA), respectively. The primary antibodies (Santa Cruz Biotechnology, Inc., Santa Cruz, CA, USA) used in this study were as follows: $\beta$-actin $(1: 1,000$, sc-7120; rabbit polyclonal), DR4 (1:1,000, sc-7863; rabbit polyclonal), DR5 (1:1,000, sc-65314; mouse monoclonal), Fas (1:1,000, sc-715; rabbit polyclonal), FasL (1:1,000, sc-957; rabbit polyclonal), TRAIL (1:500, sc-7877; rabbit polyclonal), cIAP-1 (1:1,000, sc-7943; rabbit polyclonal), cIAP-2 (1:1,000, sc-7944; rabbit polyclonal), XIAP (1:1,000, sc-11426; rabbit polyclonal), caspase-3 (1:1,000, sc-7272; mouse monoclonal), caspase-8 (1:1,000, sc-7890; rabbit polyclonal), caspase-9 (1:1,000, sc-7885; rabbit polyclonal), PARP (1:1,000, sc-7150; rabbit polyclonal), Bcl-2 (1:1,000, sc-509; mouse monoclonal), Bax (1:1,000, sc-493; rabbit polyclonal), Bid (1:500, sc-11423; rabbit polyclonal). Peroxidase-labeled donkey anti-rabbit and sheep anti-mouse immunoglobulin were purchased from Amersham Corp. All other chemicals were purchased from Sigma-Aldrich Chemical Co.

Cell culture. The 5637 human bladder cancer, Chang liver (an immortalized non-tumor cell line derived from normal liver tissue), and murine Raw 364.7 macrophage cell lines were obtained from American Type Culture Collection (Manassas, MD, USA) and maintained in RPMI-1640 medium supplemented with $10 \% \mathrm{FBS}$ and antibiotics $(100 \mu \mathrm{g} / \mathrm{ml}$ streptomycin, $100 \mathrm{U} / \mathrm{ml}$ penicillin) at $37^{\circ} \mathrm{C}$ in a humidified incubator under an atmosphere of $5 \% \mathrm{CO}_{2}$ in air. Baicalein was dissolved in dimethyl sulfoxide (DMSO) as a stock solution at $100 \mathrm{mM}$, which was then diluted with RPMI-1640 medium to the desired concentration prior to use.

Cell viability and growth assay. The 5637 cells were seeded in 6 -well plates at a density of $4.0 \times 10^{5}$ cells per well. After a $24-\mathrm{h}$ incubation, the cells were treated with various concentrations of baicalein for $24 \mathrm{~h}$. Cell viability was determined using the MTT assay. In brief, an MTT working solution $(0.5 \mathrm{mg} / \mathrm{ml})$ was added to the culture plates and incubated for $3 \mathrm{~h}$ at $37^{\circ} \mathrm{C}$. The culture supernatant was removed from the wells, and DMSO was added to dissolve the formazan crystals completely. The absorbance of each well was measured at $540 \mathrm{~nm}$ using an enzyme-linked immunosorbent assay (ELISA) reader (Molecular Devices, Sunnyvale, CA, USA). Cell growth was assessed using the trypan blue dye exclusion assay. After treatment with the indicated concentrations of the baicalein for $24 \mathrm{~h}$, the cells were trypsinized and viable cells were counted by trypan blue dye exclusion using a hemocytometer under an inverted microscope (Carl Zeiss, Jena, Germany). The effect of baicalein on cell viability and growth was assessed as the percentage of cell viability, in which the vehicle-treated cells were considered $100 \%$ viable.

Nuclear staining with DAPI. For the assessment of apoptosis, the cells were washed with ice-cold phosphate-buffered saline (PBS) and fixed with $4 \%$ paraformaldehyde in PBS for $10 \mathrm{~min}$ at room temperature. The fixed cells were washed with PBS and stained with $2.5 \mu \mathrm{g} / \mathrm{ml}$ DAPI solution for $10 \mathrm{~min}$ at room temperature. The cells were then washed twice with PBS and analyzed with a fluorescence microscope (Carl Zeiss).

DNA flow cytometric detection of apoptosis. For the quantitative assessment of the induced cell apoptosis rate, an Annexin V-FITC staining assay was performed according to the manufacturer's protocol. Briefly, the cells in each sample were stained with $5 \mu \mathrm{l}$ Annexin V-FITC and $5 \mu \mathrm{l}$ propidium iodide (PI). After incubation for $15 \mathrm{~min}$ at room temperature in the dark, the degree of apoptosis was quantified by a flow cytometer (FACSCalibur, Becton-Dickinson, San Jose, CA, USA) as a percentage of the Annexin V-positive and PI-negative cells (25). 
A<smiles>O=c1cc(-c2ccccc2)oc2cc(O)c(O)c(O)c12</smiles>

B


Figure 1. Growth inhibition of 5637 cells by treatment with baicalein. (A) Chemical structure of baicalein. (B-E) 5637, Chang liver and Raw 364.7 cells were plated into 6 -well plates at $4.0 \times 10^{5}$ cells/well and were treated with variable concentrations of baicalein. After 24-h treatment, cell viability and growth were determined by an MTT assay (B, D and E) and trypan blue dye exclusion assay (B). Data are presented as mean \pm SD values obtained from three independent experiments ( ${ }^{*} \mathrm{p}<0.05$ compared with the untreated control group).

Protein extraction and western blot analysis. After removing the media, the cells were washed with ice-cold PBS and harvested and lysed with a lysis buffer $[20 \mathrm{mM}$ sucrose, $1 \mathrm{mM}$ ethylendiaminetetraacetic acid, $20 \mu \mathrm{M}$ Tris- $\mathrm{Cl}, \mathrm{pH} 7.2,1 \mathrm{mM}$ dithiothreitol (DTT), $10 \mathrm{mM} \mathrm{KCl,} 1.5 \mathrm{mM} \mathrm{MgCl}_{2}, 5 \mu \mathrm{g} / \mathrm{ml}$ pepstatin A, $10 \mu \mathrm{g} / \mathrm{ml}$ leupeptin, and $2 \mu \mathrm{g} / \mathrm{ml}$ aprotinin] for $30 \mathrm{~min}$ at $4^{\circ} \mathrm{C}$. The supernatants were collected and protein concentration was determined using a Bio-Rad protein assay kit (Bio-Rad Laboratories, Hercules, CA, USA) according to the manufacturer's instructions. For western blotting, equal amounts of protein extracts were denatured by boiling at $95^{\circ} \mathrm{C}$ for $5 \mathrm{~min}$ in a sample buffer [0.5 M Tris- $\mathrm{HCl}, \mathrm{pH} 6.8$, $4 \%$ sodium dodecyl sulfate (SDS), $20 \%$ glycerol, $0.1 \%$ bromophenol blue, and $10 \% \beta$-mercaptoethanol] at a ratio of $1: 1$. Samples were stored at $-80^{\circ} \mathrm{C}$ or immediately used for immunoblotting. The equal cellular proteins were separated via denaturing SDS-polyacrylamide gel electrophoresis and transferred electrophoretically to nitrocellulose membranes (Amersham Corp.). The membranes were then blocked with $5 \%$ skim milk and incubated overnight at $4^{\circ} \mathrm{C}$ with primary antibodies, probed with enzyme-linked secondary antibodies for $1 \mathrm{~h}$ at room temperature, and detected using an ECL detection system.

In vitro caspase activity assay. The activities of the caspases were determined using colorimetric assay kits, which utilize synthetic tetrapeptides [Asp-Glu-Val-Asp (DEAD) for caspase-3; Ile-Glu-Thr-Asp (IETD) for caspase-8; and
Leu-Glu-His-Asp (LEHD) for caspase-9] labeled with p-nitroaniline (pNA), which is linked to the end of the caspasespecific substrate. Briefly, the baicalein-treated cells and the untreated cells were lysed in the supplied lysis buffer. The supernatants were collected and incubated with the supplied reaction buffer containing DTT and DEAD-pNA, IETD-pNA, or LEHD-pNA as substrates at $37^{\circ} \mathrm{C}$ for $2 \mathrm{~h}$ in the dark. The reactions were measured by changes in absorbance at $405 \mathrm{~nm}$ using an ELISA reader (26).

Measurement of mitochondrial membrane potential (MMP, $\left.\Delta \Psi_{m}\right)$. The MMP values were determined using the dualemission potential-sensitive probe, JC-1. Briefly, the cells were collected and incubated with $10 \mu \mathrm{M}$ of $\mathrm{JC}-1$ for $20 \mathrm{~min}$ at $37^{\circ} \mathrm{C}$ in the dark. After the JC- 1 was removed, the cells were washed with PBS to remove unbound dye, and the amount of JC-1 retained by 10,000 cells per sample was measured at 488 and $575 \mathrm{~nm}$ using a flow cytometer (27).

Detection of ROS generation. To assess the generated ROS, the cells were treated with or without $10 \mathrm{mM}$ NAC for $1 \mathrm{~h}$ before challenge with $250 \mu \mathrm{M}$ baicalein for $30 \mathrm{~min}$, and then stained with $10 \mu \mathrm{M}$ DCF-DA and incubated at $37^{\circ} \mathrm{C}$ for $30 \mathrm{~min}$ in the dark to monitor ROS production. Later, the ROS production in cells was monitored by a flow cytometer (28).

Statistical analysis. All data are presented as mean \pm standard deviation (SD). Significant differences among the groups 
A Baicalein $(\mu \mathrm{M})$

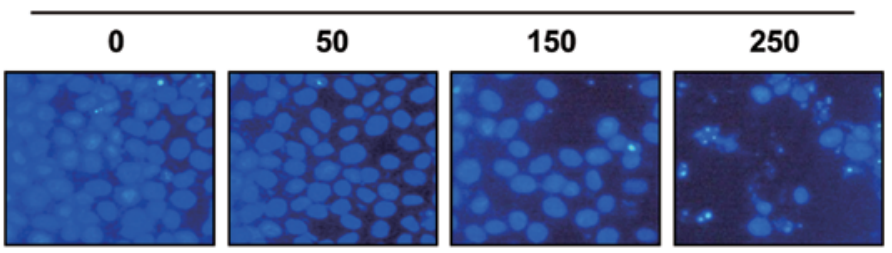

B

Baicalein $(\mu \mathrm{M})$
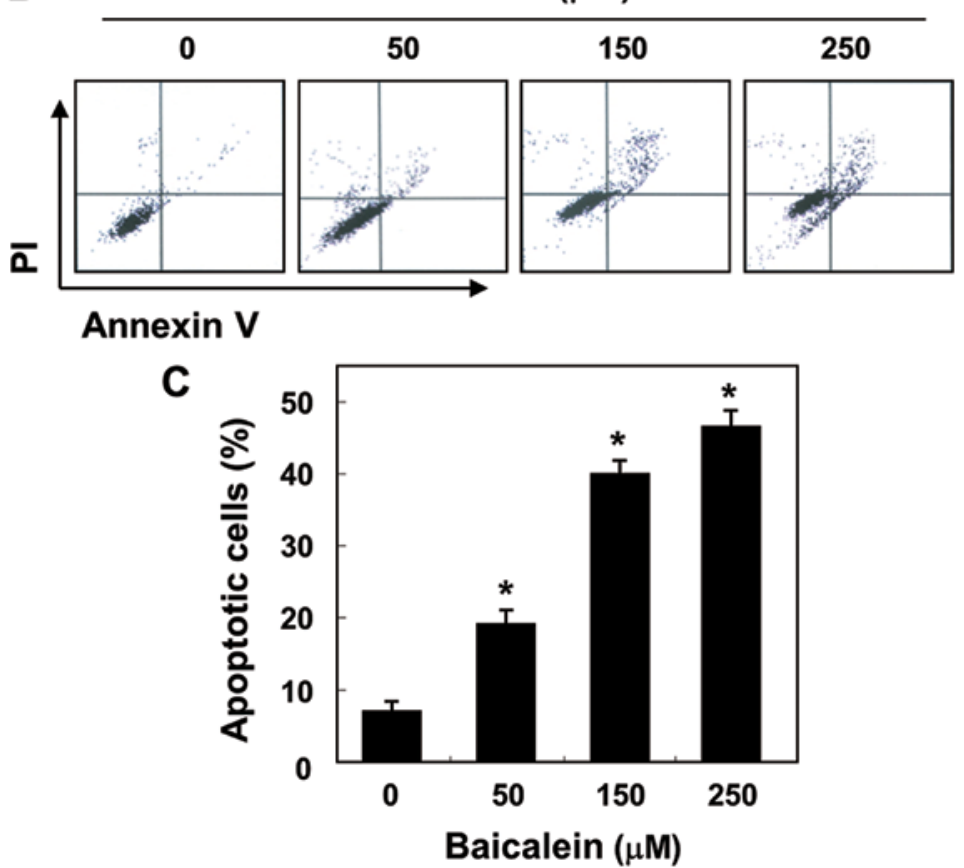

Figure 2. Induction of apoptosis by baicalein in 5637 cells. Cells were treated with the indicated concentrations of baicalein for 24 h. (A) Nuclei stained with DAPI solution were photographed with a fluorescence microscope using a blue filter; $x 400$ magnification. (B and C) To quantify the degree of cell apoptosis, the cells were stained with Annexin V-FITC and PI, and the percentage of the apoptotic cells (Annexin $\mathrm{V}^{+}$cells) were then analyzed by flow cytometric analysis. Data are presented as mean \pm SD values obtained from three independent experiments ( ${ }^{*}$ p $<0.05$ compared with the untreated control group).

were determined using the unpaired Student's t-test. A value of $\mathrm{p}<0.05$ was accepted as an indication of statistical significance. All of the data shown herein were obtained from at least three independent experiments.

\section{Results}

Effects of baicalein on the cell viability and growth in 5637 cells. To assess the effect of baicalein on 5637 cell viability and growth, equal numbers of cells were treated with various concentrations of baicalein $(50-250 \mu \mathrm{M})$ for $24 \mathrm{~h}$ and the cell viability and growth were detected by the MTT and trypan blue dye exclusion assays. As shown in Fig. 1B and C, baicalein caused dose-dependent inhibition of cell viability and growth, with a significant reduction at $150 \mu \mathrm{M}$ and an almost $60 \%$ reduction at $250 \mu \mathrm{M}$. The results of an additional experiment using Chang liver cells and Raw 264.7 macrophages, conducted to examine the effect of baicalein on the proliferation of normal cells, are shown in Fig. 1D and E. The MTT assay results indicated that baicalein concentrations $\leq 250 \mu \mathrm{M} / \mathrm{ml}$ did not induce cytotoxicity.

Induction of apoptosis by baicalein in 5637 cells. To determine whether baicalein-mediated inhibition of cell viability in 5637 cells is associated with induction of apoptosis, we examined apoptotic features by measuring the chromatin condensation of the nuclei and the amount of Annexin V-positive cells. As shown in Fig. 2A, the results of the DAPI reagent indicate that the nuclear fragmentation and chromatin condensation located in apoptotic cells were observed in baicalein-treated cells, with bright blue fluorescence. In addition, treatment with baicalein resulted in the increased accumulation of cells in the number of Annexin V-positive cells in a concentration-dependent manner (Fig. 2B and C). These findings suggest that baicalein suppresses 5637 cell viability by inducing cellular apoptosis.

Modulation of apoptosis-related genes by baicalein in 5637 cells. In order to determine which apoptosis pathway contributes to baicalein-induced apoptosis, the levels of death receptors (DRs) and corresponding proapoptotic ligands were first examined by western blot analysis. After baicalein treatment, the protein levels of Fas were not altered; however, the expression of DR4, DR5, Fas ligand (FasL), and tumor necrosis factor-related apoptosis-inducing ligand (TRAIL) were increased (Fig. 3). Next, we examined the effects of baicalein on the levels of the inhibitor of apoptosis protein (IAP) family of proteins. The results of western blotting showed that baicalein treatment resulted in a concentration-dependent decrease 


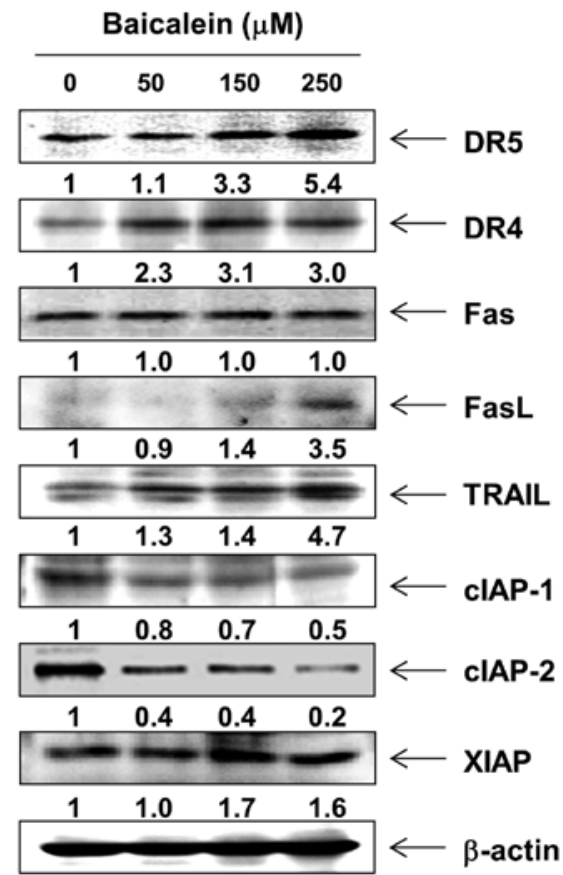

Figure 3. Effects of baicalein on the expression of DR-related and IAP family of proteins in 5637 cells. Cells were treated with the indicated concentrations of baicalein for $24 \mathrm{~h}$. Cells were lysed, and equal cellular proteins $(50 \mu \mathrm{g})$ were separated on SDS-polyacrylamide gels and transferred to nitrocellulose membranes. Membranes were probed with the indicated antibodies, and cellular proteins were visualized using an ECL detection system. $\beta$-actin was used as an internal control. The relative ratios of expression in the results of western blotting are presented at the bottom of each of the results as relative values of $\beta$-actin expression.

in the expression levels of cIAP-1 and cIAP-2, but not XIAP (Fig. 3).

Activation of caspases and cleavage of PARP by baicalein in 5637 cells. We then examined the expression levels and activities of caspases during baicalein-induced 5637 cell apoptosis. As shown in Fig. 4A, western blot analyses showed that the expression levels of pro-caspase- 3 in cells treated with baicalein were concentration-dependently downregulated and the expression levels of active-caspase- 3 were upregulated. In addition, the active forms of caspase- 8 and -9 were increased, and the levels of the pro-forms of caspase- 8 and -9 , initiator caspases of extrinsic and intrinsic apoptosis pathways, respectively, were downregulated. Under the same conditions, the in vitro activities of these caspases were measured using substrates specific to each caspase, and we found that baicalein stimulates caspase-3, -8 and -9 activities in a concentrationdependent manner (Fig. 4B). Moreover, baicalein treatment leads to progressive proteolytic cleavage of poly(ADP-ribose)polymerase (PARP), a substrate protein of active caspase-3 (Fig. 4A).

Activation of the mitochondrial apoptosis pathway by baicalein in 5637 cells. To further confirm the baicalein-induced apoptotic pathway, this study examined the effects of baicalein on the levels of Bcl-2 family expression and the MMP values. As shown in Fig. 5A, in response to baicalein treatment, the levels of proapoptotic Bax were upregulated, but those of
A

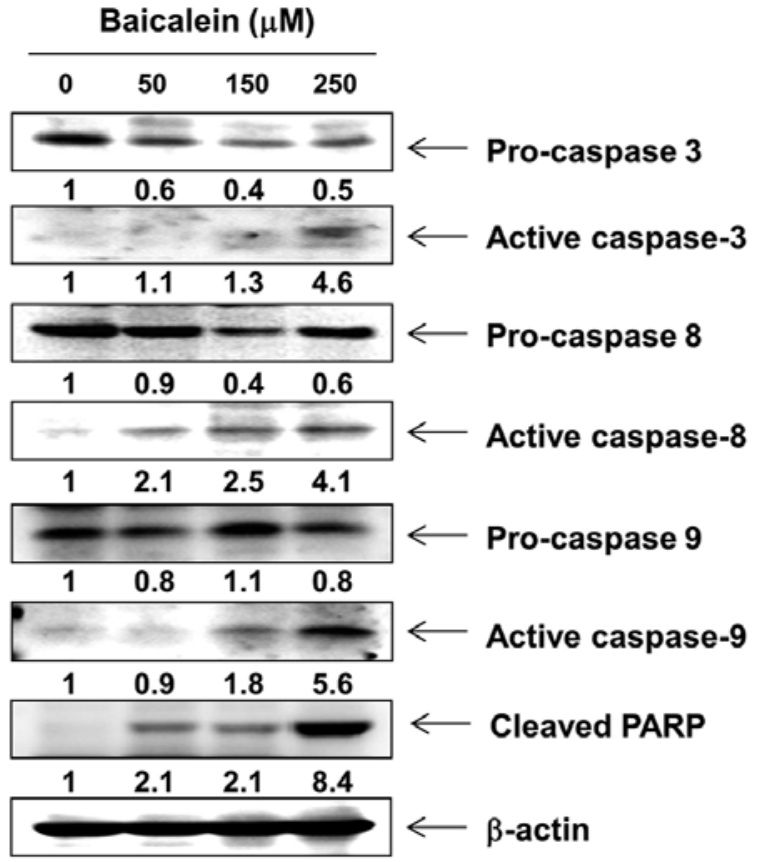

B

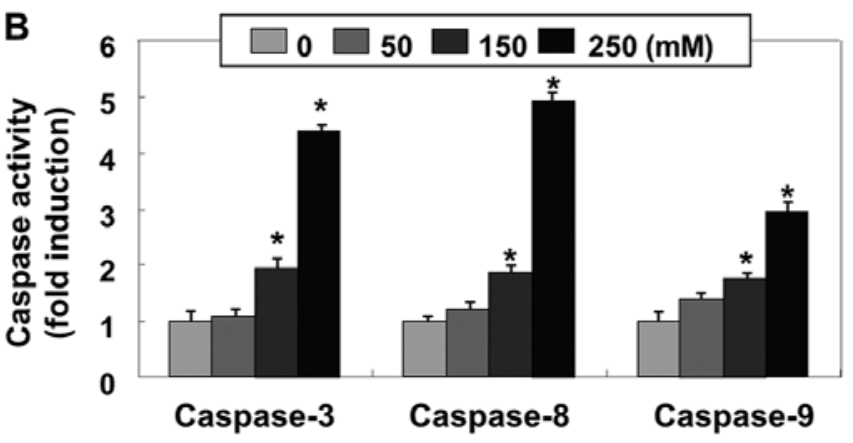

Figure 4. Activation of caspases and degradation of PARP by baicalein in 5637 cells. (A) Cells grown under the same conditions as shown in Fig. 3 were lysed, and cellular proteins $(50 \mu \mathrm{g})$ were visualized using the indicated antibodies and an ECL detection system. $\beta$-actin was used as an internal control. The relative ratios of expression in the results of western blotting are presented at the bottom of each of the results as relative values of $\beta$-actin expression. (B) Cells were lysed, and aliquots (50 $\mu$ g protein) were assayed for in vitro caspase-3, -8 and -9 activities using DEVD-pNA, IETD-pNA, and LEHD-pNA as substrates, respectively, at $37^{\circ} \mathrm{C}$ for $1 \mathrm{~h}$. The released fluorescent products were measured. Data are presented as mean \pm SD values obtained from three independent experiments $\left({ }^{*} \mathrm{p}<0.05\right.$ compared with the untreated control group).

antiapoptotic Bcl-2 were downregulated. Subsequent western blot analyses revealed progressive downregulation of total Bid protein and accumulation of truncated Bid (tBid). Moreover, baicalein treatment caused a concentration-dependent loss of MMP in comparison to the untreated control (Fig. 5B and C).

Induction of caspase-dependent apoptosis by baicalein in 5637 cells. To confirm the involvement of baicalein-induced activation of caspases, the cells were pretreated with or without z-VAD-fmk, a pan-caspase inhibitor, for $1 \mathrm{~h}$, followed by treatment with baicalein. Pretreatment with z-VAD-fmk resulted in significant prevention of the loss of MMP, expression of the active form of caspase-3, and cleavage of PARP (Fig. 6A and B). Furthermore, z-VAD-fmk also decreased the accumulation of Annexin V-FITC stained cells and increased 


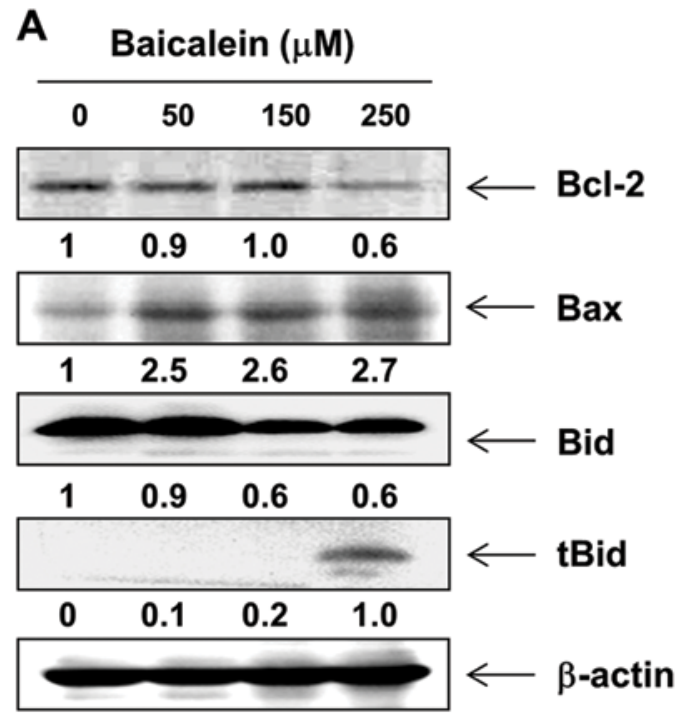

B Baicalein $(\mu \mathrm{M})$
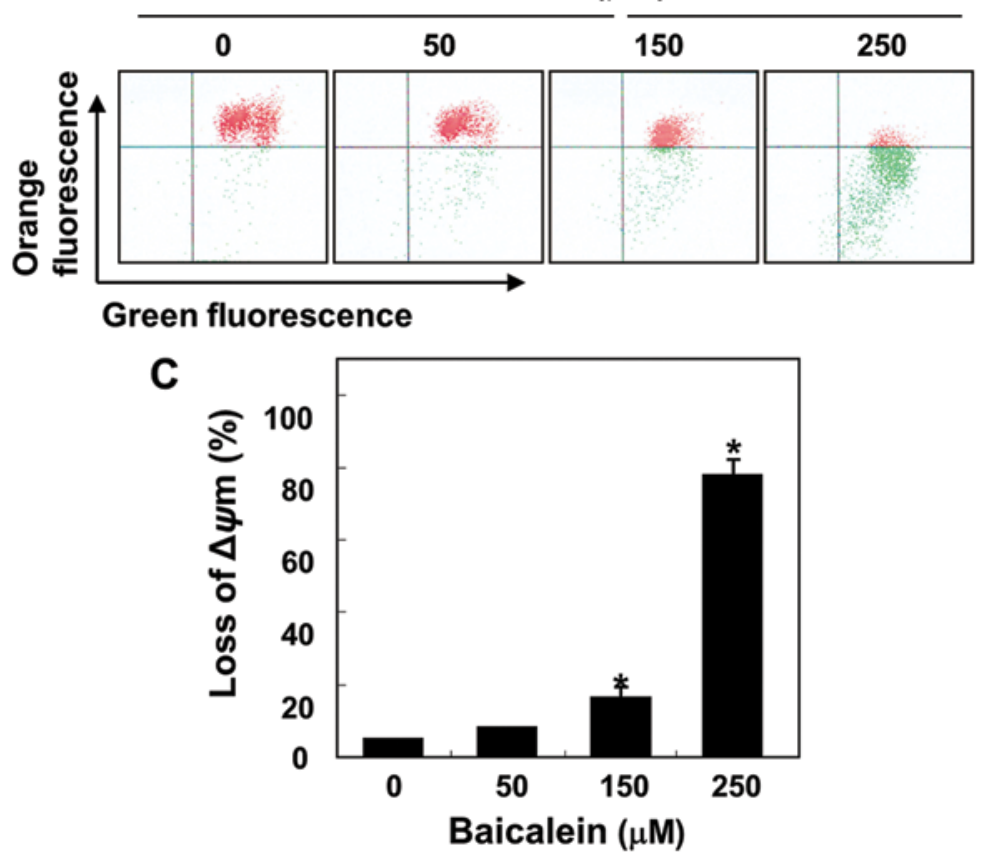

Figure 5. Effects of baicalein on the expression of Bcl-2 family of proteins and MMP values in 5637 cells. (A) Cells were treated with baicalein for $24 \mathrm{~h}$, and aliquots containing the total protein levels $(50 \mu \mathrm{g})$ were subjected to SDS-polyacrylamide gels followed by immunoblot analysis with the specific antibodies. The relative ratios of expression in the results of western blotting are presented at the bottom of each of the results as relative values of $\beta$-actin expression. (B and C) To quantify the degree of MMP loss, untreated control and baicalein-treated cells were collected and incubated with $10 \mu \mathrm{M} \mathrm{JC}-1$ for $20 \mathrm{~min}$ at $37^{\circ} \mathrm{C}$ in the dark. Cells were washed once with PBS, and MMP loss was then evaluated using a flow cytometer. Data are presented as mean \pm SD values obtained from three independent experiments ( $\mathrm{p}<0.05$ compared with the untreated control group).

A

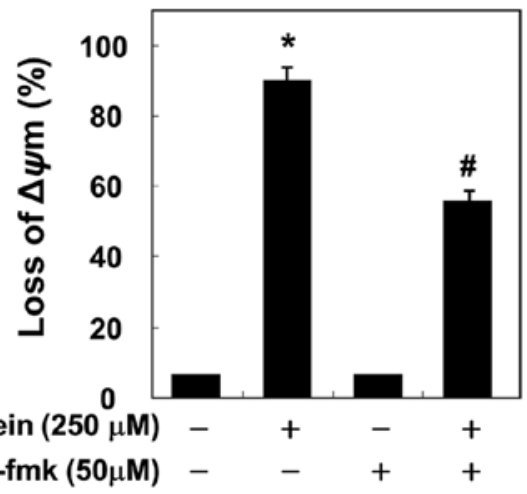

C

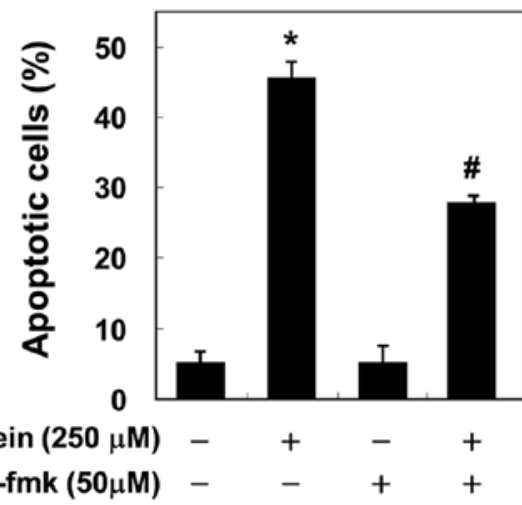

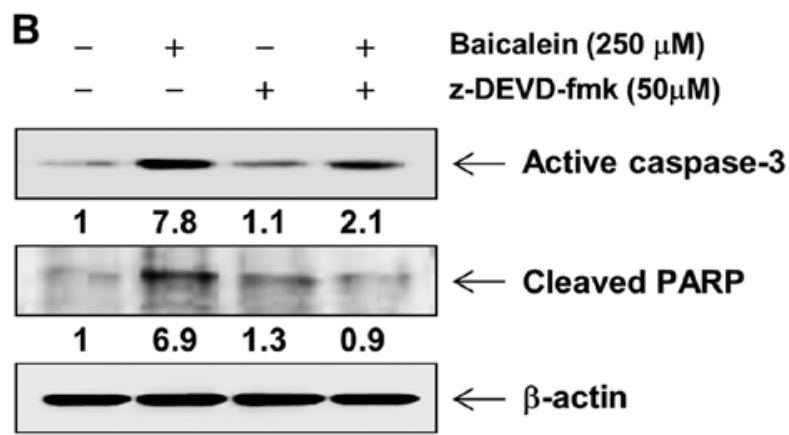

D

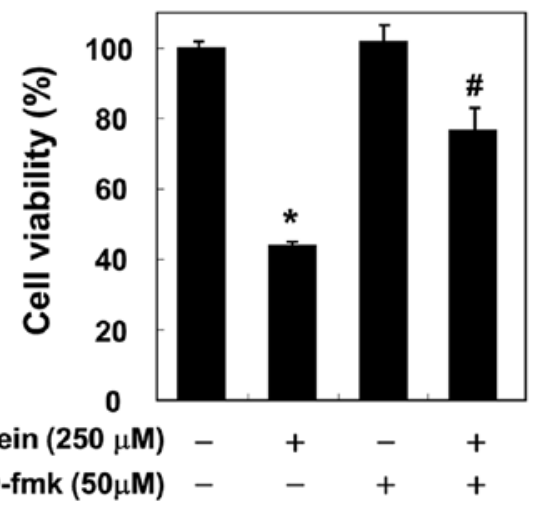

Figure 6. Inhibition of baicalein-induced apoptosis by pan-caspase inhibitor in 5637 cells. Cells were pretreated for $1 \mathrm{~h}$ with or without $50 \mu \mathrm{M} \mathrm{z}$-VED-fmk and treated with 5637 for an additional 24 h. (A) Cells were stained with JC-1, and MMP loss was evaluated using a flow cytometer. (B) Cells were lysed, and equal cellular proteins $(50 \mu \mathrm{g})$ were separated on SDS-polyacrylamide gels and transferred to nitrocellulose membranes. Membranes were probed with anti-caspase- 3 and anti-PARP antibodies, and cellular proteins were visualized using an ECL detection system. $\beta$-actin was used as an internal control. The relative ratios of expression in the results of western blotting are presented at the bottom of each of the results as relative values of $\beta$-actin expression. (C) The percentage of apoptotic cells (Annexin $\mathrm{V}^{+}$cells) was analyzed using flow cytometric analysis. (D) Cell viability was determined by an MTT assay. Each point represents the mean $\pm \mathrm{SD}$ of three independent experiments ( $\mathrm{p}<0.05$ vs. untreated control; ${ }^{\#} \mathrm{p}<0.05$ vs. baicalein-treated cells). 

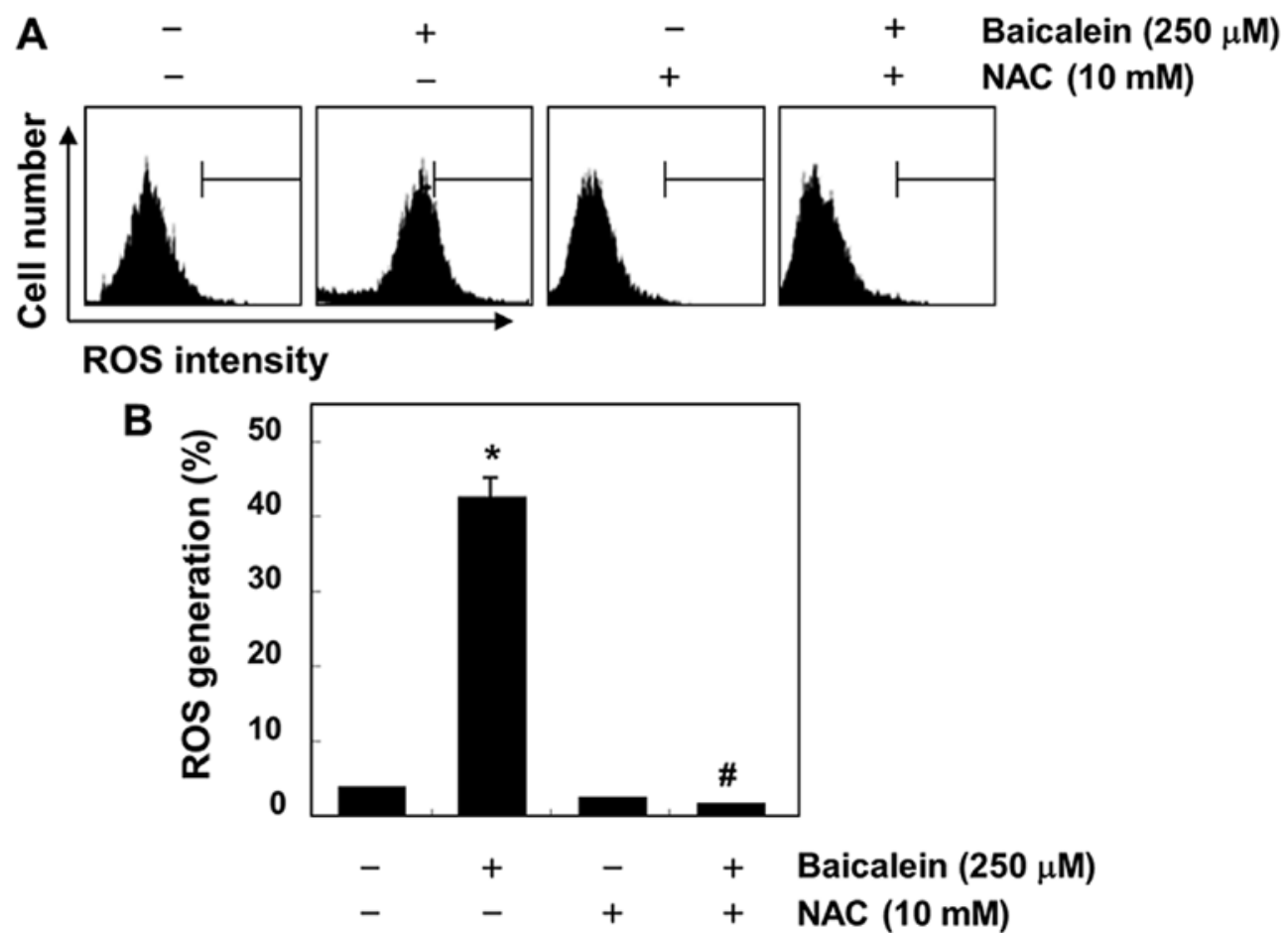

Figure 7. Generation of ROS by baicalein in 5637 cells. Cells were treated with or without $10 \mathrm{mM}$ NAC for $1 \mathrm{~h}$ before challenge with $250 \mu \mathrm{M}$ baicalein for $30 \mathrm{~min}$ and were then stained with DCF-DA and incubated at $37^{\circ} \mathrm{C}$ for $30 \mathrm{~min}$. (A) At each time-point, fluorescent intensity was measured using a flow cytometer. (B) Each point represents the mean $\pm \mathrm{SD}$ of three independent experiments ( ${ }^{*} \mathrm{p}<0.05$ vs. untreated control; ${ }^{*} \mathrm{p}<0.05$ vs. baicalein-treated cells).

A



C

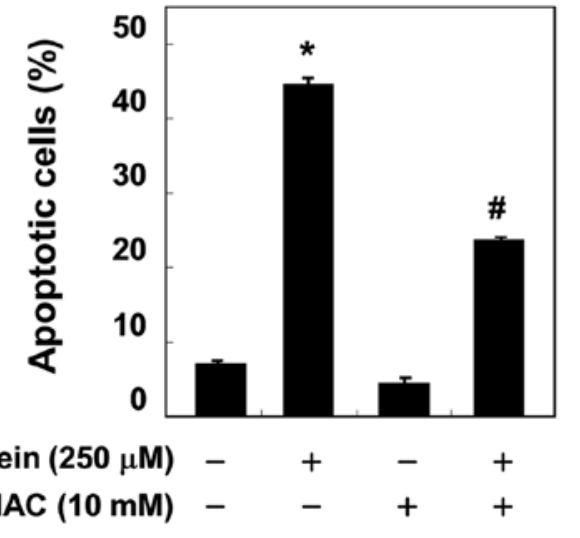

Baicalein $(250 \mu \mathrm{M})-\quad+-+$ NAC (10 mM) - - + +
B $-\quad+\quad-\quad+\quad$ Baicalein $(250 \mu \mathrm{M})$ $-\quad+\quad+\quad$ NAC (10 mM)
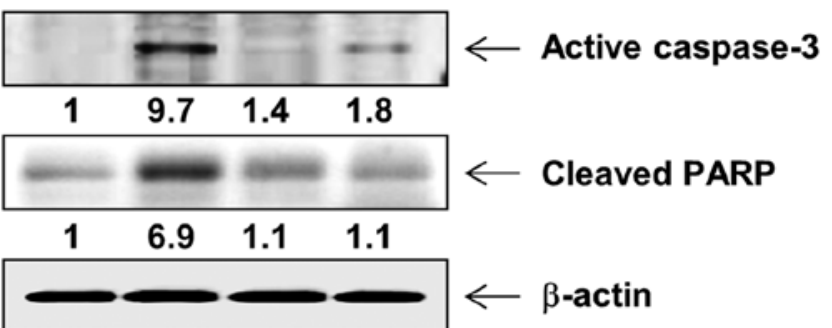

D

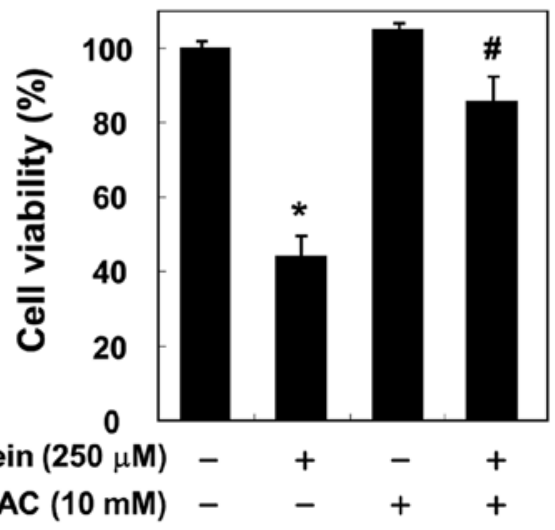

Figure 8. Induction of ROS-mediated apoptosis by baicalein in 5637 cells. Cells were pretreated without or with NAC (10 mM) for $1 \mathrm{~h}$ before treatment with baicalein $(250 \mathrm{mM}$ ) for $24 \mathrm{~h}$. (A and C) Loss of MMP and the percentage of apoptotic cells was measured using a flow cytometer. (B) Protein expression was detected using anti-caspase-3 and anti-PARP antibodies, and cellular proteins were visualized using an ECL detection system. $\beta$-actin was used as an internal control. The relative ratios of expression in the results of western blotting are presented at the bottom of each of the results as relative values of $\beta$-actin expression. (D) Cell viability was determined by an MTT assay. Each point represents the mean \pm SD of three independent experiments $\left({ }^{*} \mathrm{p}<0.05\right.$ vs. untreated control; ${ }^{\mathrm{p}}<0.05$ vs. baicalein-treated cells). 
cell viability in the presence of baicalein (Figs. 6C and D). These results provide evidence of baicalein-induced apoptotic cell death in association with the activation of caspases in 5637 cells.

Induction of ROS-dependent apoptosis by baicalein in 5637 cells. Because the generation of intracellular ROS may be related to mitochondrial dysfunction and the induction of apoptosis in various cell types, we further investigated whether baicalein could stimulate ROS generation in 5637 cells. As shown in Fig. 7, the maximal generation of ROS was observed at 30-min treatment with baicalein; however, baicalein-induced ROS generation was effectively blocked by the antioxidant NAC. In addition, the loss of MMP, activation of caspase- 3 and cleavage of PARP induced by baicalein were significantly attenuated by pretreatment with NAC (Fig. 8A and B). We also observed that baicalein-induced apoptosis and reduction of cell viability were markedly reduced by pretreatment with NAC (Fig. 8C and D), suggesting that baicalein induces apoptosis via ROS-dependent caspase activation mechanisms in 5637 cells.

\section{Discussion}

In this study, we investigated whether baicalein induces cell death in human bladder cancer 5637 cells and sought to identify the mechanisms related to apoptosis. The findings demonstrate that baicalein concentration-dependently inhibits cell viability and induces apoptosis, as measured by chromatin condensation of the nuclei and Annexin V-stained cells, which are the hallmark features of apoptosis. The findings also suggest that baicalein stimulates caspase-dependent extrinsic and intrinsic apoptosis pathways in 5637 cells. Furthermore, our results show that baicalein-induced ROS generation and activation of caspases are significantly suppressed by pretreatment with NAC, an antioxidant, indicating that ROS are the upstream regulators of caspase activation during baicaleininduced apoptosis.

Apoptosis is the rigorous, active, and orderly process of cell death, and dysregulated apoptosis is considered to induce a number of pathological conditions, including cancer $(29,30)$. In general, apoptosis may be initiated through two major pathways, the extrinsic (DR-mediated) pathway and the intrinsic (mitochondrial-mediated) pathway, both of which involve the activation of caspases $(31,32)$. The products of several genes have been demonstrated to be critical in the regulation of these two pathways, including caspase cascades, Bcl-2, and IAP family members. In the extrinsic pathway, the binding of extracellular death ligands to their cell-surface DRs leads to the activation of caspase-8 $(32,33)$. On the other hand, the intrinsic pathway is activated by the release of proapoptotic factors such as cytochrome $c$ from the mitochondria to the cytosol, following the loss of inner-mitochondrial-membrane integrity and activation of caspase-9. Moreover, the extrinsic pathway can crosstalk to the intrinsic pathway through the caspase-8-mediated cleavage of Bid, a member of the Bcl-2 family of proteins, which ultimately amplify the intrinsic apoptotic pathway $(33,34)$. Therefore, the induction of apoptosis is an important target for cancer therapy, and agents that target the apoptosis pathway without affecting normal cells play crucial roles as potential drug targets in cancer treatment.

In this study, we examined various aspects of the mechanisms of apoptosis induction by baicalein in 5637 cells, and we found that baicalein activates initiator caspases (caspase-8 and -9) of the extrinsic and intrinsic pathways as well as downstream effector caspase-3 (Fig. 4), which is associated with the degradation of PARP, a hallmark of apoptosis and substrates of activated caspase (35). Although the levels of Fas remained unchanged after baicalein treatment, baicalein considerably increased the levels of DR4, DR5, FasL, and TRAIL. Baicalein also partially downregulated the IAP family of proteins, such as cIAP-1 and cIAP-2 (Fig. 3), which reportedly block apoptosis due to their function as direct inhibitors by binding to and inhibiting several caspases $(36,37)$. In addition, baicalein increased the expression levels of proapoptotic Bax and inhibited antiapoptotic Bcl-2, which was associated with a dose-dependent loss of MMP and a decline in intact Bid occurring concurrently with a pronounced increase of tBid (Fig. 5). Therefore, our current data suggest that baicalein induces Bid truncation, leading to the release of proapoptotic factors to the cytosol by enhancing mitochondrial dysfunction and ultimately to the induction of apoptosis in 5637 cells. However, blocking caspase activity by pretreating the cells with z-VAD-fmk, a pan-caspase inhibitor, significantly attenuated baicalein-induced apoptosis and growth inhibition (Fig. 6). Therefore, the data suggest that baicalein-induced apoptosis in 5637 cells is caspase-dependent and that both the intrinsic and the extrinsic pathways are activated by baicalein.

Previous research has determined that the enhancement of ROS production is associated with the apoptotic response induced by various chemotherapeutic agents in a variety of cell types $(38,39)$. Mitochondria are both the source and target of ROS generation, and damaged mitochondria can release more ROS $(40,41)$. Previous studies have suggested that ROS and mitochondria may mediate apoptosis induction under both physiological and pathological conditions $(38,39)$. ROS can cause the loss of MMP by activating mitochondrial permeability transition and can induce apoptosis by releasing apoptogenic proteins to the cytosol $(42,43)$. Moreover, a number of studies have noted that the proapoptotic potential of some anticancer agents is highly correlated with the generation of ROS from mitochondria $(38,40)$, indicating that the inhibition of ROS accumulation can serve as an effective strategy for the treatment of cancers. Thus, we further investigated whether the generation of intracellular ROS is necessary for baicalein-induced apoptosis, and we found that ROS levels were markedly increased in baicalein-treated 5637 cells within $30 \mathrm{~min}$ (Fig. 7). However, baicalein-induced ROS generation in cells that were co-cultured with NAC, a commonly used reactive oxygen intermediate scavenger (44), was effectively blocked. These results indicate that if ROS is a crucial factor in the induction of apoptosis in baicaleintreated 5637 cells, a ROS scavenger must abrogate apoptosis. As shown in Fig. 8, NAC alone had no effect on the MMP, caspase- 3 expression, and PARP cleavage; however, the presence of NAC suppressed the baicalein-induced loss of MMP, upregulation of active-caspase-3, and degradation of PARP. Furthermore, the blocking of ROS generation significantly 
prevented baicalein-induced apoptosis as well as loss of cell viability. Taken together, these findings suggest that an increase in ROS is required for the occurrence of baicaleininduced apoptosis in 5637 cells.

In conclusion, the results of this study demonstrate that baicalein triggers apoptosis of human bladder cancer 5637 cells through the activation of both the intrinsic caspase pathway and the death-receptor-mediated extrinsic pathway and that the activation of caspases is responsible for the mediation of baicalein-induced apoptosis, which requires ROS generation upstream of the disruption of MMP and activation of caspase. The data emphasize the key role of ROS in apoptosis induced by baicalein in 5637 cells and indicate a positive correlation between ROS and mitochondrial events leading to apoptosis. Although these findings indicate that ROS play a pivotal role in the regulation of baicalein-induced apoptosis in 5637 cells, further studies are warranted to investigate the direct effect of baicalein using an in vivo model.

\section{Acknowledgements}

This study was supported by the National Research Foundation of Korea (NRF) grant funded by the Korean Government (MISP) (no. 2015R1A2A2A01004633) and the Functional Districts of the Science Belt support program, Ministry of Science, ICT and Future Planning.

\section{References}

1. Lei AQ, Cheng L and Pan C-X: Current treatment of metastatic bladder cancer and future directions. Expert Rev Anticancer Ther 11: 1851-1862, 2011.

2. van Kessel KE, Zuiverloon TC, Alberts AR, Boormans JL and Zwarthoff EC: Targeted therapies in bladder cancer: An overview of in vivo research. Nat Rev Urol 12: 681-694, 2015.

3. Donato F, Boffetta P, Fazioli R, Aulenti V, Gelatti U and Porru S Bladder cancer, tobacco smoking, coffee and alcohol drinking in Brescia, northern Italy. Eur J Epidemiol 13: 795-800, 1997.

4. Wu X, Ros MM, Gu J and Kiemeney L: Epidemiology and genetic susceptibility to bladder cancer. BJU Int 102B: 1207-1215, 2008.

5. Pal SK, Milowsky MI and Plimack ER: Optimizing systemic therapy for bladder cancer. J Natl Compr Canc Netw 11: 793-804 2013.

6. Feuerstein MA and Goenka A: Quality of life outcomes for bladder cancer patients undergoing bladder preservation with radiotherapy. Curr Urol Rep 16: 75, 2015.

7. Fridlender M, Kapulnik Y and Koltai H: Plant derived substances with anti-cancer activity: From folklore to practice. Front Plant Sci 6: 799, 2015.

8. Cragg GM and Newman DJ: Plants as a source of anti-cancer agents. J Ethnopharmacol 100: 72-79, 2005.

9. Nagai T, Yamada $\mathrm{H}$ and Otsuka Y: Inhibition of mouse liver sialidase by the root of Scutellaria baicalensis. Planta Med 55: 27-29, 1989.

10. Li-Weber M: New therapeutic aspects of flavones: The anticancer properties of Scutellaria and its main active constituents Wogonin, Baicalein and Baicalin. Cancer Treat Rev 35: 57-68, 2009.

11. Huang Y, Tsang SY, Yao X and Chen ZY: Biological properties of baicalein in cardiovascular system. Curr Drug Targets Cardiovasc Haematol Disord 5: 177-184, 2005.

12. Li C, Lin G and Zuo Z: Pharmacological effects and pharmacokinetics properties of Radix Scutellariae and its bioactive flavones. Biopharm Drug Dispos 32: 427-445, 2011.

13. Firuzi O, Miri R, Tavakkoli M and Saso L: Antioxidant therapy: Current status and future prospects. Curr Med Chem 18: 3871-3888, 2011.
14. He X, Wei Z, Zhou E, Chen L, Kou J, Wang J and Yang Z: Baicalein attenuates inflammatory responses by suppressing TLR4 mediated NF- $\mathrm{B}$ and MAPK signaling pathways in LPS-induced mastitis in mice. Int Immunopharmacol 28: 470-476, 2015.

15. Xiao JR, Do CW and To CH: Potential therapeutic effects of baicalein, baicalin, and wogonin in ocular disorders. J Ocul Pharmacol Ther 30: 605-614, 2014.

16. Cheng YH, Li LA, Lin P, Cheng LC, Hung CH, Chang NW and Lin C: Baicalein induces G1 arrest in oral cancer cells by enhancing the degradation of cyclin D1 and activating AhR to decrease $\mathrm{Rb}$ phosphorylation. Toxicol Appl Pharmacol 263: 360-367, 2012.

17. Zheng YH, Yin LH, Grahn TH, Ye AF, Zhao YR and Zhang QY: Anticancer effects of baicalein on hepatocellular carcinoma cells. Phytother Res 28: 1342-1348, 2014.

18. Lee HZ, Leung HW, Lai MY and Wu CH: Baicalein induced cell cycle arrest and apoptosis in human lung squamous carcinoma CH27 cells. Anticancer Res 25A: 959-964, 2005.

19. Aryal P, Kim K, Park PH, Ham S, Cho J and Song K: Baicalein induces autophagic cell death through AMPK/ULK1 activation and downregulation of mTORC1 complex components in human cancer cells. FEBS J 281: 4644-4658, 2014.

20. Kim DH, Hossain MA, Kang YJ, Jang JY, Lee YJ, Im E, Yoon JH, Kim HS, Chung HY and Kim ND: Baicalein, an active component of Scutellaria baicalensis Georgi, induces apoptosis in human colon cancer cells and prevents AOM/DSS-induced colon cancer in mice. Int J Oncol 43: 1652-1658, 2013.

21. Chung H, Choi HS, Seo EK, Kang DH and Oh ES: Baicalin and baicalein inhibit transforming growth factor- $\beta 1$-mediated epithelial-mesenchymal transition in human breast epithelial cells. Biochem Biophys Res Commun 458: 707-713, 2015.

22. Chen J, Li Z, Chen AY, Ye X, Luo H, Rankin GO and Chen YC: Inhibitory effect of baicalin and baicalein on ovarian cancer cells. Int J Mol Sci 14: 6012-6025, 2013.

23. Rushworth SA and Micheau O: Molecular crosstalk between TRAIL and natural antioxidants in the treatment of cancer. Br J Pharmacol 157: 1186-1188, 2009.

24. Kim HJ, Park C, Han MH, Hong SH, Kim GY, Hoon Hong S, Deuk Kim N and Choi YH: Baicalein induces caspase-dependent apoptosis associated with the generation of ROS and the activation of AMPK in human lung carcinoma A549 cells. Drug Dev Res 77: 73-86, 2016.

25. Park MH and Han JS: Padina arborescens extract protects high glucose-induced apoptosis in pancreatic $\beta$ cells by reducing oxidative stress. Nutr Res Pract 8: 494-500, 2014.

26. Kim SJ, Ho Hur J, Park C, Kim HJ, Oh GS, Lee JN, Yoo SJ, Choe SK, So HS, Lim DJ, et al: Bucillamine prevents cisplatininduced ototoxicity through induction of glutathione and antioxidant genes. Exp Mol Med 47: e142, 2015.

27. Kim YS, Li XF, Kang KH, Ryu B and Kim SK: Stigmasterol isolated from marine microalgae Navicula incerta induces apoptosis in human hepatoma HepG2 cells. BMB Rep 47: 433-438, 2014.

28. Song JL, Choi JH, Seo JH, Kil JH and Park KY: Antioxidative effects of fermented sesame sauce against hydrogen peroxideinduced oxidative damage in LLC-PK1 porcine renal tubule cells. Nutr Res Pract 8: 138-145, 2014.

29. Fadeel B and Orrenius S: Apoptosis: A basic biological phenomenon with wide-ranging implications in human disease. J Intern Med 258: 479-517, 2005.

30. Sayers TJ: Targeting the extrinsic apoptosis signaling pathway for cancer therapy. Cancer Immunol Immunother 60: 1173-1180, 2011.

31. MacKenzie SH and Clark AC: Targeting cell death in tumors by activating caspases. Curr Cancer Drug Targets 8: 98-109, 2008.

32. Brenner D and Mak TW: Mitochondrial cell death effectors. Curr Opin Cell Biol 21: 871-877, 2009.

33. Hensley P, Mishra M and Kyprianou N: Targeting caspases in cancer therapeutics. Biol Chem 394: 831-843, 2013.

34. Jin Z and El-Deiry WS: Overview of cell death signaling pathways. Cancer Biol Ther 4: 139-163, 2005.

35. Kaufmann SH, Desnoyers S, Ottaviano Y, Davidson NE and Poirier GG: Specific proteolytic cleavage of poly(ADP-ribose) polymerase: An early marker of chemotherapy-induced apoptosis. Cancer Res 53: 3976-3985, 1993.

36. Danson S, Dean E, Dive C and Ranson M: IAPs as a target for anticancer therapy. Curr Cancer Drug Targets 7: 785-794, 2007. 
37. de Graaf AO, de Witte T and Jansen JH: Inhibitor of apoptosis proteins: New therapeutic targets in hematological cancer? Leukemia 18: 1751-1759, 2004

38. Kardeh S, Ashkani-Esfahani S and Alizadeh AM: Paradoxical action of reactive oxygen species in creation and therapy of cancer. Eur J Pharmacol 735: 150-168, 2014.

39. Matés JM, Segura JA, Alonso FJ and Márquez J: Oxidative stress in apoptosis and cancer: An update. Arch Toxicol 86: 1649-1665, 2012.

40. Boneh A: Regulation of mitochondrial oxidative phosphorylation by second messenger-mediated signal transduction mechanisms. Cell Mol Life Sci 63: 1236-1248, 2006.
41. Fleury C, Mignotte B and Vayssière JL: Mitochondrial reactive oxygen species in cell death signaling. Biochimie 84: 131-141, 2002.

42. Fulda $\mathrm{S}$ and Debatin KM: Extrinsic versus intrinsic apoptosis pathways in anticancer chemotherapy. Oncogene 25: 4798-4811, 2006.

43. Ghobrial IM, Witzig TE and Adjei AA: Targeting apoptosis pathways in cancer therapy. CA Cancer J Clin 55: 178-194, 2005.

44. Zafarullah M, Li WQ, Sylvester J and Ahmad M: Molecular mechanisms of N-acetylcysteine actions. Cell Mol Life Sci 60: 6-20, 2003. 